Revista Científica General José María Córdova, Bogotá, Colombia, enero-junio, 2016

DERECHOS HUMANOS Y DERECHO INTERNACIONAL HUMANITARIO

Vol. 14, Núm. 17 pp. 129-148 Issn 1900-6586

Cómo citar este artículo: Cabrera García, V.E., Cuervo Ríos, J.C., Martínez Castro, Z. \& Cabrera Campos, M.A. (2016, enero-junio). Estrategias para afrontar el divorcio en personas de las Fuerzas Militares de Colombia. Rev. Cient. Gen. José María Córdova 14(17), 129-148

\title{
Estrategias de afrontamiento frente al divorcio en personas de las Fuerzas Militares de Colombia*
}

Recibido: 30 de agosto de 2015 • Aceptado: 25 de noviembre de 2015

\section{Confrontation strategies against divorce in people of the Military Forces of Colombia}

Stratégies d'affrontement contre le divorce dans personnes des Forces militaires de la Colombie

Estratégias de enfrentamento contra o divórcio em pessoas das Forças Militares da Colômbia

\author{
Victoria Eugenia Cabrera García ${ }^{a}$ \\ Juan Carlos Cuervo Ríos ${ }^{b}$ \\ Zoraida Martínez Castro ${ }^{c}$ \\ Miguel Angel Cabrera Campos ${ }^{2}$
}

*Artículo de investigación, proyecto: "Impacto psicosocial del divorcio en el Ejército de Colombia", Maestría en Familia, Universidad de la Sabana.

a Universidad de La Sabana, Chía, Colombia. Coordinadora de investigaciones del Instituto de la Familia de la Universidad de La Sabana. Comentarios a: victoria.cabrera@unisabana.edu.co

b Universidad de La Sabana, Chía, Colombia. Magíster en Asesoría familiar gestión de programas para la familia, Universidad de La Sabana, Chía, Colombia. juancuri@unisabana.edu.co

c Universidad de La Sabana, Chía, Colombia. Magíster en Asesoría familiar gestión de programas para la familia, Universidad de La Sabana, Bogotá, Colombia. zoraidamaca@unisabana.edu.co

d Universidad de La Sabana, Chía, Colombia. Magíster en Asesoría familiar gestión de programas para la familia, Universidad de La Sabana, Chía, Colombia. miguelcabcam@unisabana.edu.co 
Resumen. El propósito del presente estudio es analizar las estrategias para afrontar el divorcio o separación, empleadas por las personas que pertenecen al Ejército Nacional y la Armada Nacional de Colombia, o porquienes han sido sus esposas o esposos. Participaron 124 personas, hombres y mujeres, militares y civiles entre los 20 y 60 años, que cumplieron con los criterios seleccionados para la muestra, como estar divorciados por un periodo mayor de seis meses y menor de tres años, con mínimo un hijo. Estas estrategias se evaluaron con el inventario de respuestas de Afrontamiento (CRI-A) para población Adulta de Moos (1993), traducido y adaptado por Isabel Mikulic y Melina Crespi, 1998. Los resultados muestran el predominio del empleo de estrategias de afrontamiento en función a la adaptación de las personas y su proceso de estrés en su vida cotidiana.

Palabras claves: Divorcio, Estrategias de Afrontamiento, Fuerzas Militares de Colombia, impacto psicosocial.

Abstract. The purpose of this study is to analyze the coping strategies used by people from the Army and National forces of Colombia or their ex-spouses, during their divorce or separation process. Participants were 124 men and women, militaries and civilians between the ages of 20 and 60 years, who achieved the criteria selected for the sample such as, being divorced for a period longer than six months and less than three years and also, with at least one child. Coping strategies were assessed with the inventory of coping responses (CRI-A) for adults, designed by Moos in 1993, translated and adapted by Isabel Mikulic and Melina Crespi in 1998. Results show the predominance of the use of coping strategies according to the people's adaptation and the stress process that they face in their daily lives.

Keywords: Divorce, Coping strategies, Military Forces of Colombia, psychosocial impact.

Résumé. Le objectif de cette étude est d'analyser les stratégies de confrontation utilisés contre divorce ou de séparation, employées par des personnes qui appartenir aux forces de l'Armée et des Forces Militaires de la Colombie, ou ceux qui ont été leurs épouses ou maris. L'étude a impliqué 124 personnes, hommes et femmes, militaires et civils entre les âges de 20 et 60 ans, qui ont accompli les critères sélectionner pour l'prélèvements comme étant divorcé pour une période de plus de six mois et moins de trois ans, avec au moins un enfant. Les stratégies d'affrontement ont été évalués à l'inventaire de confronter les réponses (CRI-A) pour les adultes faites par Moos en 1993, traduit et adapté par Isabel Mikulic et Melina Crespi en 1998. Les résultats montrent la prédominance de la utilisation des stratégies de confrontation, avec la finalité de l'adaptation du peuple et le processus de stress auxquels ils sont confrontés dans leur vie quotidienne.

Mots-clés: divorce, stratégies de confrontation, Forces Militaires de la Colombie, impact psychosocial.

Resumo. O objetivo do presente estudo é analisar as estratégias de enfrentamento utilizadas em situação de divórcio ou separaçáo, empregadas por membros do Exército Nacional e da Marinha Nacional da Colômbia, ou por aqueles que hajam sido seus cônjuges. Participaram124 pessoas, homens e mulheres, militares e civis, com idades entre 20 e 60 anos, que atenderam aos requisitos de seleção da amostra, como estarem divorciados por um período maior que 6 meses e menos que 3 anos, com no mínimo um filho. As estratégias de enfrentamento foram avaliadas com o inventário de respostas de enfrentamento (CRI-A) para população adulta de Moos, 1993, traduzido e adaptado por Isabel Mikulic e Melina Crespi, 1998. Os resultados mostram a predominância do emprego de estratégias de enfrentamento em relação à adaptação das pessoas e ao processo de estresse que as mesmas enfrentam em suas vidas diárias.

Palavras-chave: Divórcio, Estratégias de Enfrentamento, Forças Armadas da Colômbia, Impacto Psicosocial. 


\section{Introducción}

El concepto occidental de la unión matrimonial o conyugal lleva implícito ideas sobre sus beneficios, en particular lo relacionado con el bienestar, estabilidad emocional, económica, apoyo, cuidado mutuo y reciprocidad entre los miembros de la pareja, (Gager, C. \& Hohmann-Marriott, B., 2006, pp. 5-42), lo que cobra aun más importancia cuando las relaciones de pareja constituyen una de las relaciones más intensas que se pueden establecer entre los seres humanos, el vínculo de mayor significación fuera de la familia de origen (Maureira, F., 2011, p. 322).

Sin embargo, cuando los conflictos se hacen cada vez más frecuentes y difíciles de solucionar por los cónyuges o miembros de la pareja, el divorcio o la separación de cuerpos es una de las soluciones más recurrentes. Si bien es cierto que eventualmente el conflicto se considera necesario, dado que este se puede entender como un factor de crecimiento, cuya resolución implica un trabajo orientado a la obtención de un nuevo equilibrio más estable y superior que el anterior (Cabrera, Guevara \& Barrera 2006, p. 116), no obstante, estos conflictos no siempre desembocan en situaciones de crecimiento familiar, sino más bien en el divorcio.

Varios investigadores han analizado la necesidad de estudiar las modalidades de afrontamiento en grupos de sujetos que se hallan ante estresores específicos (Carver, C., Pozo, C., Harris, S., Noriega, V., Scheier, M., Robinson, E., Ketcham, A., Moffat, F. \& Clark, K., 1993, pp. 375390). La población militar es una población en alto riesgo de experimentar eventos estresores a nivel personal y familiar como el divorcio, debido en parte a su misión, como también a la alta movilidad geográfica que experimentan sus miembros, por lo cual vale la pena estudiar fenómenos en este contexto, específicamente los que afectan a la familia, máxime cuando la escasa literatura existente invita a desarrollar estudios con el fin de contribuir con el conocimiento y avance de la ciencia en el contexto militar colombiano y específicamente en la familia militar.

En este orden de ideas, esta investigación se propuso como objetivo conocer cuáles son las estrategias de afrontamiento utilizadas por las personas en medio del proceso de divorcio. Específicamente, se pretendió indagar cuáles son las más y menos utilizadas por los hombres y por las mujeres, y cuáles las más y menos utilizadas por personas militares y civiles.

\section{Divorcio}

Según Profamilia, en Colombia los divorcios se han venido incrementado, más específicamente, se han triplicado en los últimos treinta años, lo cual incide negativamente en el bienestar y la calidad de vida del grupo familiar (Monroy 2007; Zamudio \& Rubiano, 1994; citados en: Jiménez, Macías, \& Amaris, 2012, pp. 99-112).

El proceso de divorcio normalmente pone en marcha numerosos eventos que las personas experimentan como estresantes. Estos factores de estrés, a su vez, aumentan el riesgo de presentar resultados negativos a nivel emocional y conductual tanto en los adultos como en los nińos. La gravedad y la duración de estos resultados negativos varía de una persona a otra, dependiendo de la presencia de una variedad de factores de protección y ajuste exitosos que se producen en la medida en que los individuos experimentan algunos síntomas relacionados con el divorcio, pero pueden funcionar bien en la nueva familia, el trabajo o la escuela, y han desarrollado una 
identidad y estilo de vida que ya no está ligada al anterior matrimonio (Kitson, G. C. \& Morgan, L. A., 1990, pp. 913-924).

Las diversas afectaciones que genera el divorcio han sido materia de estudio de diferentes áreas del conocimiento, en diferentes momentos de la historia; en ese sentido lo que sucede hoy día no dista mucho de lo descrito en su tiempo por los padres de la iglesia Tertuliano y San Jerónimo, cuando revisaron la decadencia de la familia a la luz de fenómenos como el descenso de la natalidad, falta de interés en la vida familiar, elevado porcentaje de la delincuencia juvenil y debilidad de los lazos familiares entre otros (Zimmerman, C. \& Uranga, E., 1946, p. 154).

A nivel mundial, en cuanto a divorcios, se presenta un patrón más o menos generalizable entre los países del mundo occidental, que va de niveles más altos de divorcio en los países socialmente más desarrollados a niveles más bajos en los países con niveles menores de desarrollo, de acuerdo con los datos que presenta el reporte de la División de Población de las Naciones Unidas para el año 2004 (Ojeda, N. \& González, E., 2008, p. 113).

El creciente fenómeno que supone el aumento en las estadísticas de divorcios en Colombia, se refleja en los datos suministrados por la Superintendencia de Notariado y Registro, al presentar un aumento del 14\% entre el 2010 y el 2011 y del 17\% entre el 2011 y 2012. Sin embargo, encontramos en el mismo informe que para el primer trimestre del año 2013, el número de divorcios registrados en las notarías disminuyó en $6.5 \%$ comparado con el mismo periodo del año inmediatamente anterior.

En concordancia con la descrita tendencia a la baja, en un estudio realizado entre los años 2011 y 2013 por el área de Investigación de la Dirección de Familia y Asistencia Social del Ejército de Colombia se observa a más de 12.000 efectivos, entre oficiales y suboficiales, que el número de personal divorciado es del 1,3\%, (DIFAM, 2014, p.8) número inferior al 2\% de divorcios en Colombia, aun cuando esta es la tasa más baja a nivel latinoamericano (Lippman, L., Wilcox , B. \& Ryberg, R., 2013, p. 31).

Sin embargo, es importante revisar detenidamente estos datos, por el mismo carácter dinámico del fenómeno, esto referido a las disoluciones conyugales que no han sido legalizadas por una autoridad civil y que, por lo mismo, no son registradas en las estadísticas vitales y frecuentemente son declaradas de manera dudosa en los censos de población (Ojeda, N. \& González, E., 2008, p. 112).

El divorcio surge a causa de expectativas sobre el matrimonio que no se logran satisfacer en la práctica, provocando que los beneficios actuales de permanecer casado, sean menores que las nuevas expectativas de beneficios provenientes de volver a ser soltero o de contraer matrimonio con otra persona (Verbel, C., 1991, pp. 1-6).

En contraste, también encontramos que la pérdida de la pareja suele ser un acontecimiento traumático, con importantes efectos adversos en el bienestar físico y psicológico de las personas que lo padecen. A pesar de que podría establecerse un cierto paralelismo entre la pérdida de la pareja por muerte (viudedad) y la separación o divorcio, hay indicios de que, debido a la naturaleza de la pérdida, es más difícil adaptarse a estos últimos. A diferencia de la viudedad, el divorcio es un proceso voluntario, plagado de sentimientos ambivalentes (Yárnoz-Yaben, S., 2013, pp. 1-11; Aldous , J. \& Ganey, R., 1999, pp.155-180; Aseltine Jr., R. H. \& Kessler, R. C., 1993, pp. $237-$ 251). En este orden de ideas, es interesante explorar cuáles son las estrategias de afrontamiento utilizadas por las personas que se divorcian en medio de este proceso. 


\section{Estrategias de afrontamiento}

A través de la historia se ha intentado comprender la dinámica que vive el ser humano cuando se ve inmerso en situaciones que le generan estrés, desbordantes, y que busca manejar a través de ciertas estrategias (Amarís, Madariaga, Valle \& Zambrano, 2013, pp. 123-145).

El afrontamiento se define y comprende "aquellos procesos cognitivos y conductuales constantemente cambiantes que se desarrollan para mejorar las demandas externas y/o internas que son evaluadas como desbordantes de los recursos del individuo" (Folkman, S., Lazarus, R. S., Dunkel-Schetter, C., DeLongis, A. \& Gruen, R. J., 1986, p. 992). Por consiguiente, las estrategias de afrontamiento se refieren a todos aquellos pensamientos, reinterpretaciones, conductas, etc., que el individuo despliega en búsqueda de obtener mejores resultados en una situación particular generadora de estrés, ya sea para obtener una regulación en la respuesta emocional (afrontamiento dirigido a las emociones) y/o manipular o alterar el problema (afrontamiento dirigido a la conducta).

En concordancia, las características y naturaleza de los sucesos a los que se enfrentan los sujetos influyen en la disponibilidad y movilización de recursos, así como también en las modalidades de afrontamiento de los mismos. La literatura refiere que existen dos tipos de estrategias de afrontamiento:

- Estrategias de afrontamiento centradas en el problema: clasificadas como de aproximación: la persona se centra en hacer frente a la situación, buscando soluciones al problema que ha provocado la disonancia cognitiva. Hay una búsqueda deliberada de solución, de recomposición del equilibrio, roto por la presencia de la situación estresante.

- Estrategias de afrontamiento centradas en la emoción: clasificadas como evitación cuando la persona busca la regulación de las consecuencias emocionales activadas por la presencia de la situación estresante.

Algunas investigaciones han estudiado la importancia del apoyo social como estrategia de afrontamiento, por ejemplo, la influencia de la familia de origen y algunas redes sociales, es decir, la participación en reuniones, clubes y organizaciones, se relacionó significativamente con la sensación de que las cosas estarían mejor, con una importante disminución de los síntomas depresivos y ansiosos (Kunz, 1996, pp. 3-4; Vollrath, M., Alnæs R., \& Torgersen R., 1994, pp. 727-736; Veiel, H., Kühne, C., Brill, G. \& Ihle, W. 1992, pp. 415-427).

Por otro lado, factores asociados al género influyen en la manera de afrontar procesos de separación: "Las problemáticas que se generan ante la disolución de la unión conyugal dependen en parte a los patrones socio-culturales y modelos ideo-afectivos, [...] la intensidad de las emociones, el grado de apego y las estrategias de afrontamiento son diferentes en varones y en mujeres" (Jiménez, Y. V., 2003, pp. 1-16).

También se ha demostrado que las estrategias de afrontamiento ante un divorcio relacionadas con la religiosidad o espiritualidad moderan los efectos negativos de experiencias de un evento traumático (Zukerman, G. \& Korn, L., 2013, pp. 1676-1690). 
Adicionalmente, McCubbin, Cauble y Patterson (1982) advierten que los procesos de afrontamiento no solo se presentan de forma individual, también aparecen como mediadores en el ámbito social. De esta forma, al interior del grupo familiar se despliega el uso de estrategias para afrontar ya sea crisis normativas o no-normativas, con el fin de mantener, y/o recuperar el equilibrio y garantizar el bienestar de sus miembros (McCubbin, McCubbin, Thompson, Sae-Young \& Allen, 1997, pp. 2-11; Hernández, 1991, citado en Amaris, Madarriaga, valle \& Zambrano 2013, pp. 123-145).

Con el fin de evaluar las estrategias de afrontamiento, se han desarrollado y validado instrumentos, como es el caso del Inventario de Respuestas de Afrontamiento, cuyo autor es Rudolf H. Moos (1993), prueba que permite analizar la interacción entre el individuo y su entorno, así como los recursos del individuo, revisando estrategias de afrontamiento de tipo aproximación o evitación (Endler \& Parker, 1990, pp. 844-854; Mikulic \& Crespi, 2008, p. 307).

En tal sentido, se ha observado que sucesos estresantes, tales como enfermedades físicas, tienden a promover mayor cantidad de respuestas de afrontamiento, activo conductual, y los debidos a relaciones interpersonales como el divorcio, más de afrontamiento focalizado en la emoción, todo esto depende de la severidad del estresor (Holahan, C., Moos, R. \& Brennan, P., 1997, pp. 918-928).

\section{Familias en el contexto militar}

Concretamente, la familia militar posee particularidades frente al resto de familias que conforman nuestra sociedad, específicamente en lo referido a la predominancia del liderato materno en el manejo del hogar, donde los varones, por la misión de su trabajo, fungen como "padres presentes-ausentes" (De Zubiría, M., 2010, p. 1), dados los largos periodos de tiempo que regularmente están por fuera del seno de su familia.

Sin embargo, contario a lo que muchos piensan, la familia militar se ha identificado como una institución relativamente estable, como lo documentan las citadas estadísticas (DIFAM, 2014).

En concordancia con esto, las acciones que en materia de familia se han venido implementando en los últimos años reflejan la necesidad de trabajar por la estabilidad de las parejas en las que al menos uno de sus miembros es militar, siguiendo modelos como el del ejército estadounidense (Alyssa, J., Jay, S., Stephen, W., Bradley, N., Joseph , P. \& Charles , C., 2010, pp. 101-109), donde los líderes militares hacen todo lo posible para ofrecer servicios de apoyo a las familias y en especial a las parejas, con particular atención a las esposas de militares, mitigando eventualmente efectos negativos en la salud mental familiar. Según Martínez (2013, p.7), encontramos lo siguiente:

Durante los últimos veinticinco años, Colombia ha venido sufriendo una transformación en la conformación de los hogares [...] evidente en mayores separaciones y divorcios. La ocurrencia de estos divorcios motiva al investigador a reflexionar sobre sus impactos psicosociales, ampliamente estudiados por las diferentes ciencias, ubicando a los estudiosos en familia y específicamente en familia militar en la necesidad de determinar a través de investigaciones la realidad de la familia en función al medio militar (Martínez, 2013, p. 7). 
De manera consonante con los riesgos descritos, las Fuerzas Militares y específicamente el Ejército Nacional de Colombia, puso en funcionamiento la primera dependencia a nivel de jefatura denominada "Jefatura de Familia y Asistencia Social", la cual, más allá de la misión particular de defensa nacional, ha entendido la necesidad de fortalecer estos espacios de responsabilidad social y familiar, en función del fortalecimiento de la familia como espacio de crecimiento, socialización y optimización integral de los hombres y mujeres que fungen como soldados. El modelo planteado por el Ejército en temas de familia ha sido exitosamente replicado por otras Fuerzas como la Armada Nacional, la Fuerza Aérea y la Policía Nacional.

De la mano con esta iniciativa, se ha apostado por darle sustento a las acciones que en temas de familia se adelantan, a través de procesos de investigación que soporten las decisiones estratégicas que afectarán a la familia de nuestros efectivos; todo alineado con el proceso de transformación, que actualmente se gesta en la Fuerza, mediante objetivos estratégicos como "propiciar desarrollos intelectuales y doctrinales que fortalezcan el proceso de transformación del Ejército y orienten su implementación" (Ciro, A., 2014, p. 21).

En función de los antecedentes reflejados en la literatura consultada, surge la siguiente cuestión: ¿cuáles son las estrategias de afrontamiento del divorcio utilizadas por las parejas con al menos un militar como uno de sus miembros?

Este estudio pretende despejar este interrogante, pues, en los últimos años, en las Fuerzas Militares, específicamente del Ejército y la Armada de Colombia, se ha hecho énfasis en la austeridad del gasto y en la maximización de los recursos existentes, especialmente el humano, en busca de eficiencia en todos los procesos, meta que se ve afectada por la ocurrencia de divorcios, pues no solo se ve disminuida la productividad laboral del uniformado, quien por este evento pierde concentración en su misión particular, sino que se generan gastos indirectos para la Institución como, por ejemplo, las asesorías profesionales (psicosociales y jurídicas) que se requieren a manera de orientación, mayormente en eventos de divorcio y pareja: a corte diciembre de 2013, se cuentan solo en la Dirección de Familia y Asistencia Social de Ejército más de 50.000 consultas atendidas en temas de familia, las cuales son impartidas por profesionales, de planta o contratados con recursos de la Institución y del Estado colombiano (DIFAM, 2014, p. 26).

\section{Preguntas de investigación}

Con base en esta revisión teórica y empírica, las preguntas que orientaron el presente estudio son: ¿cuáles son las estrategias de afrontamiento más y menos utilizadas en las Fuerzas Militares frente a la ocurrencia del divorcio?, ¿cuáles son las estrategias de afrontamiento más y menos utilizadas por hombres y mujeres de las Fuerzas Militares frente a la ocurrencia del divorcio?, ¿cuáles son las estrategias de afrontamiento más y menos utilizadas por personal civil y militar frente a la ocurrencia del divorcio? 


\section{Método}

\section{Tipo de investigación}

Este estudio es de corte transversal, univariado descriptivo, en el que se analizaron las diferencias entre medias.

\section{Participantes}

Participaron en este estudio 124 personas de las cuales el 48\% (60) son uniformadas de las Fuerzas Militares de Colombia, y el 52\% (64) son civiles que están separados o divorciados de personal uniformado de la Armada Nacional o del Ejército de Colombia, entre 20 y 60 ańos $M=39 \mathrm{DS}=6,56$, de los cuales 57 fueron hombres $M=36 \mathrm{DS}=4,76$ y 67 mujeres $M=33 \mathrm{DS}=5,27$. La muestra fue tomada de diferentes ciudades, como Bogotá, Medellín, Barranquilla, Pasto, entre otras ciudades de Colombia.

Para el presente estudio se ha seleccionado una muestra intencional, de 124 participantes de los cuales 105 pertenecen al Ejercito y 19 a la Armada Nacional de Colombia, los criterios de inclusión son los siguientes: tener más de seis meses de divorciados o separados y menos de tres años en la misma condición, así como tener mínimo un hijo de dicha relación. Se contempló este lapso de tiempo debido a que los momentos más estresantes del divorcio se presentan durante este tiempo aproximadamente. Se excluyeron del estudio aquellas personas separadas que no tenían hijos, y que siendo civiles estén separados de otro civil.

\section{Procedimiento}

Se llevaron a cabo los siguientes pasos:

1. Un grupo de psicólogos y trabajadores sociales se entrenaron en la aplicación del instrumento CRI-A. Estos profesionales, por su ubicación geográfica a lo largo y ancho de Colombia, fueron capacitadas mediante video conferencia. La aplicación se hizo en las diferentes unidades donde laboran estos profesionales, junto con los militares y civiles examinados, el instrumento se sistematizó y los participantes lo diligenciaron en línea, en los lugares apartados donde no hubo acceso a la red, el instrumento se hetero-aplicó de manera escrita para ser ingresado a la base de datos posteriormente.

2. La participación de militares y civiles fue voluntaria, lograda mediante la identificación de personas con los criterios de inclusión en la población objeto de la Dirección de Familia del Ejército, y, de la misma manera, en la dependencia homóloga en la Armada de Nacional de Colombia para asuntos familiares. Las familias fueron contactadas de manera personal. La aplicación de instrumentos se realizó por separado a cada participante, previa concertación de una cita. Se firmó el consentimiento informado y se registró su número telefónico para verificar cualquier información necesaria.

3. Los análisis de datos se han realizado con el programa estadístico SPSS versión 21. 


\section{Instrumento}

Se empleó la adaptación argentina del Inventario de Respuestas de Afrontamiento para adultos CRI-A de autoría del señor Rudolf H. Moos, Ph. D., traducida y adaptada por la doctora Isabel María Mikulic, se analizó la consistencia interna del inventario, así como su validez factorial. El coeficiente alpha del instrumento fue elevado (.97) y los coeficientes de las escalas específicas, adecuados. El uso de este tipo de inventarios, por su versatilidad, permite utilizarlo como instrumento de investigación y de evaluación de personas, pudiendo abarcar aspectos cuantitativos y cualitativos, elemento concomitante con el diseño mixto escogido para este ejercicio (García, M., 2003, pp. 1-29), su principal ventaja es que permite consultar a una población amplia de una manera rápida y económica.

El inventario de Respuestas de Afrontamiento para adultos CRI-A tiene como objetivo la evaluación de los diferentes tipos de repuestas de afrontamiento ante situaciones estresantes de la vida, combinando dos dimensiones: el foco del afrontamiento (en el problema o en la emoción) y el método del afrontamiento (cognitivo o conductual). La aplicación del instrumento se realizó en versión impresa y digital, inicialmente los participantes diligenciaron el consentimiento informado y los datos sociodemográficos solicitados como género, edad, situación laboral, nivel educativo, procedencia, entre otros, posteriormente seleccionaban y describían el estresor o problema relacionado con la separación o divorcio de su pareja, para luego diligenciar el inventario de respuestas de afrontamiento, compuesto de 48 ítems que se responden en una escala de tipo Likert cuyas opciones varían entre " $0=$ Nunca" y " $5=$ casi siempre". El Inventario se compone de 48 ítems que evalúan ocho respuestas de afrontamiento, así:

- Análisis lógico (AL): Esta escala se refiere a los intentos cognitivos de comprender y prepararse mentalmente para enfrentar un estresor y sus consecuencias, cuenta con seis preguntas como: ¿Trató de encontrarle alguna explicación o significado a esa situación?

- Revalorización positiva (R): se refiere a los intentos cognitivos de construir y reestructurar un problema en un sentido positivo mientras se acepta la realidad de una situación, cuenta con 6 preguntas como: ¿Pensó cómo podrían estos sucesos cambiar su vida en un sentido positivo?

- $\quad$ Búsqueda de orientación y apoyo (BA): referida a los intentos conductuales de buscar información, apoyo y orientación, cuenta con seis preguntas como: ¿Buscó la ayuda de personas o de grupos con los mismos problemas?

- Resolución de problemas (RP): referida a los intentos conductuales de realizar acciones conducentes directamente al problema, cuenta con seis preguntas como: ¿Trató de resolver los problemas, al menos en dos formas diferentes?

- Evitación cognitiva (EC): referida a los intentos cognitivos de evitar pensar en el problema de forma realista, cuenta con seis preguntas como: ¿Evitó pensar en el problema, aun sabiendo que en algún momento debería pensar en él?

- Aceptación/Resignación (A): referida a los intentos cognitivos de reaccionar al problema aceptándolo, cuenta con seis preguntas como: ¿Aceptó los problemas, porque pensó que nada se podía hacer?

- Búsqueda de gratificaciones alternativas (BG): referida a los intentos conductuales de involucrarse en actividades substitutivas y crear nuevas fuentes de satisfacción, cuenta 
con seis preguntas como: ¿Volvió al trabajo o a otras actividades que lo ayudaran a enfrentar las cosas?

- Descarga emocional (DE): referida a los intentos conductuales de reducir la tensión expresando sentimientos negativos, cuenta con seis preguntas como: ¿Hizo algo arriesgado tratando de tener una nueva oportunidad?

Las sub-escalas de AL, R, RP y BA se consideran estrategias de aproximación y las sub-escalas de EC, A, BG y DE se definen como estrategias de evitación. Desde el punto de vista del método de afrontamiento, las estrategias que pertenecen a la dimensión cognitiva son AL, R, EC y A, mientras que RP, BA, BG y DE corresponden a la dimensión conductual. En cada uno de estos dos grupos, las primeras dos escalas evalúan las respuestas cognitivas, y la tercera y cuarta escalas, las respuestas conductuales del afrontamiento, (Mikulic, I. \& Crespi, M., 2008, p.307).

\section{Resultados}

A lo largo de este ejercicio investigativo se realiza un acercamiento y descripción a las estrategias de afrontamiento utilizadas frente al divorcio, por parejas donde al menos uno de los cónyuges es militar, para lo cual se ha realizado un estudio de corte transversal, los resultados se analizaron teniendo en cuenta una estadística descriptiva, univariada, mediante análisis de diferencias de medias. Se ha tomado como base el análisis de frecuencias y porcentajes presentadas en las tablas y gráficos con sus respectivos estadísticos. Véase las tablas 1 a la 2 y figuras 1 a la 4 .

\section{Estrategias de afrontamiento frente al divorcio utilizadas en las Fuerzas Militares en general}

Respecto a las estrategias de afrontamiento más utilizadas por los participantes, "Rezo pidiendo ayuda o fuerza" $\mathrm{M}=2.52$ (0.86) y "Decidió lo que quería hacer y trató firmemente de conseguirlo" $\mathrm{M}=2.41$ (1.10), se encontró que éstas pertenecen predominantemente a estrategias de aproximación y a la dimensión conductual (Ver tabla 1).

Tabla 1. Estrategias de afrontamiento más usadas por los participantes en general.

\begin{tabular}{lcll}
\hline \multicolumn{1}{c}{ Escala } & Media & Dimensión & Estrategia \\
\hline Búsqueda de guía y apoyo & 2.52 & Conductual & Aproximación \\
\hline Solución de problemas & 2.41 & Conductual & Aproximación \\
\hline Solución de problemas & 2.39 & Conductual & Aproximación \\
\hline Evitación cognitiva & 2.32 & Cognitiva & Evitación \\
\hline Solución de problemas & 2.31 & Conductual & Aproximación \\
\hline
\end{tabular}

Fuente: Elaboración propia

En contraste, las escalas de afrontamiento menos utilizadas por los participantes "Se mantuvo alejado de la gente en general" $(\mathrm{M}=.80)$ y "Trató de negar lo serio que eran en realidad los problemas" ( $\mathrm{M}=.96)$, pertenecen a descarga emocional, relacionadas con las estrategias de evitación, contrario 
a lo observado en la tabla uno, con respecto a la determinación de los participantes por resolver las situaciones de estrés, situación esperable dado el entrenamiento de los militares frente a la resolución de conflictos y la influencia de este entrenamiento en las personas que conforman su familia, predominantemente en procesos de modelamiento por observación (ver tabla 2).

Tabla 2. Estrategias de afrontamiento menos usadas por los participantes en general

\begin{tabular}{lcll}
\hline \multicolumn{1}{c}{ Escala } & Media & Dimensión & Estrategia \\
\hline Descarga emocional & .80 & Conductual & Evitación \\
\hline Búsqueda de apoyo & .90 & Conductual & Aproximación \\
\hline Evitación cognitiva & .96 & Cognitiva & Evitación \\
\hline Descarga emocional & 1.02 & Conductual & Evitación \\
\hline Descarga emocional & 1.14 & Conductual & Evitación \\
\hline
\end{tabular}

Fuente: Elaboración propia

\section{Estrategias de afrontamiento más y menos utilizadas según el sexo}

Con respecto a las estrategias más utilizadas por hombres y mujeres, se encontró que los hombres utilizan predominantemente las estrategias de aproximación en la dimensión conductual, con conductas como "Decidió lo que quería hacer y trató finalmente de conseguirlo" $(\mathrm{M}=2.49)$ donde hacen frente a la situación, buscando soluciones al problema de forma deliberada para alcanzar la recomposición del equilibrio roto, por la presencia de situaciones estresantes. En contraste, las mujeres del estudio reportaron como frecuencia más alta, conductas como "Rezó pidiendo ayuda o fuerza" ( $(\mathrm{M}=2.70)$, donde utilizaron las estrategias de evitación en la dimensión cognitiva, en la escala aceptación resignación.

Sin embargo, las medias de las dos siguientes escalas para las mujeres, muestran, en contraste, la utilización de estrategias de afrontamiento similares a los hombres del estudio. Aquí cabe recordar lo dicho en relación con que estas mujeres, en su mayoría, son ex-esposas de militares y, por consiguiente, las que históricamente llevaban la batuta en las decisiones familiares (ver figura 1).

Las estrategias de afrontamiento menos usadas por los hombres del estudio se reflejan en la evitación de conductas como "Gritó como forma de desahogarse" $(\mathrm{M}=0.65)$, que son de tipo evitación en la dimensión conductual en la escala de descarga emocional. Esto refleja que en pocas ocasiones ellos le dan la espalda a los problemas que provocan desequilibrio personal o familiar, apartado también hay coincidencia entre hombres y mujeres, pues la estrategia que más alto puntaje reporta para las mujeres es "Mantenerse alejadas de la gente" $(M=0.84)$ también es de evitación en la dimensión conductual, al experimentar dificultades relacionadas con el divorcio (ver figura 2). 


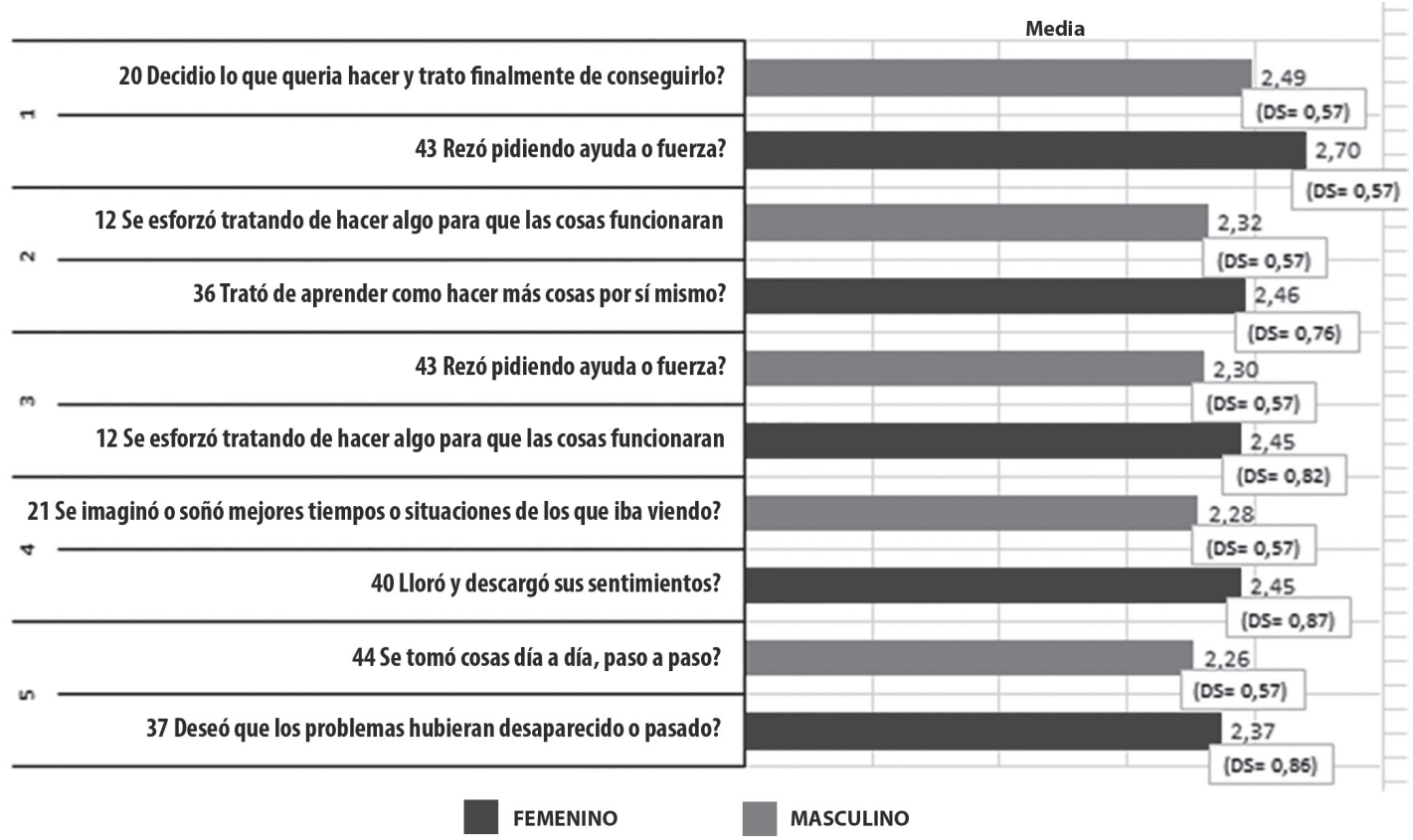

Figura 1. Estrategias de afrontamiento más usadas por hombres y mujeres.

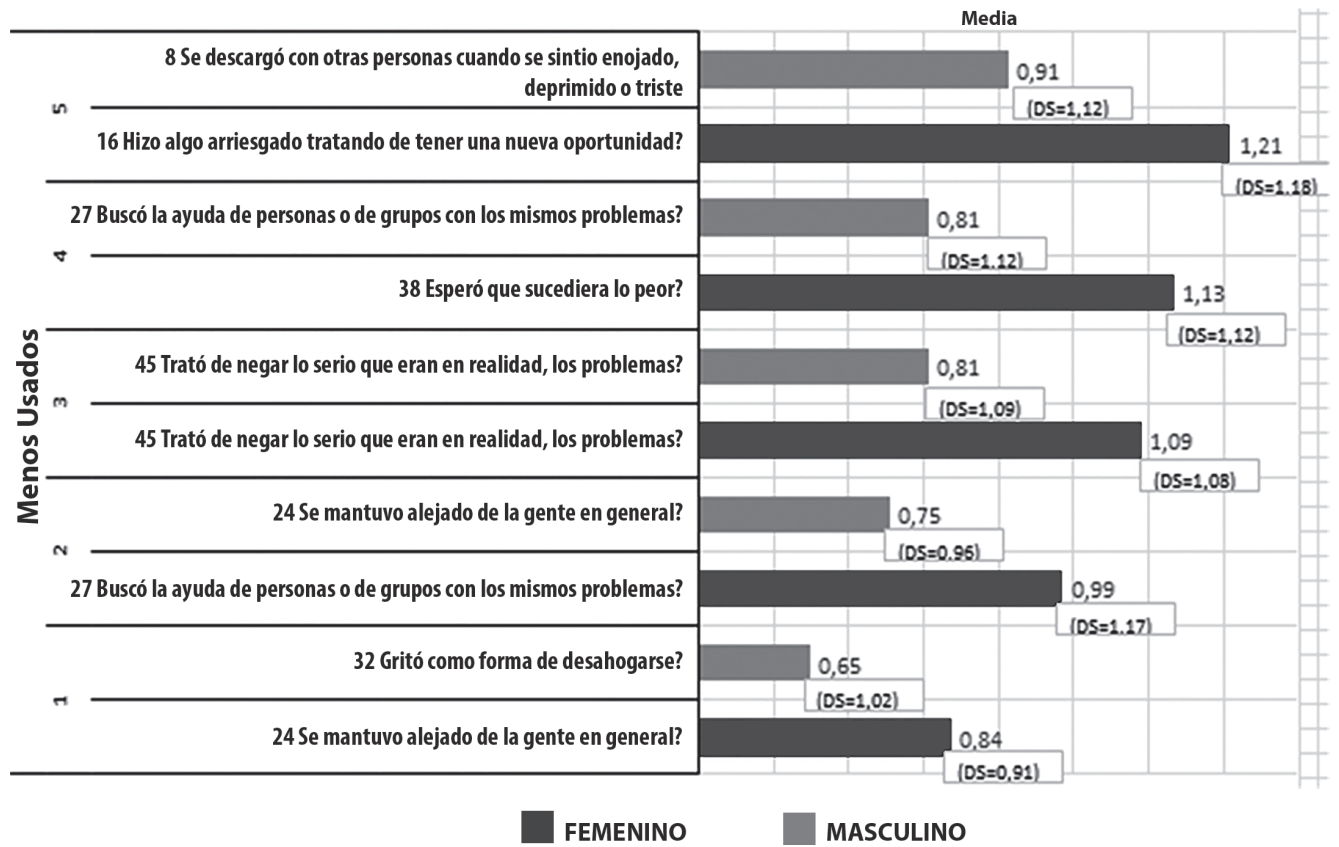

Figura 2. Estrategias de afrontamiento menos usadas por hombres y mujeres. 


\section{Estrategias de afrontamiento más y menos utilizadas por civiles y militares}

$\mathrm{Al}$ evaluar las estrategias de afrontamiento más usadas por los militares, es importante tener en cuenta dos aspectos: en primer lugar, la estrategia de afrontamiento más usada, "Decidió lo que quería hacer y trató firmemente de conseguirlo" $(\mathrm{M}=2.50)$ corresponde a las estrategias de aproximación en la dimensión conductual, le siguen "Se imaginó o soñó mejores tiempos o situaciones de los que estaba viviendo" ( $\mathrm{M=2.40)}$ y "Volvió al trabajo o a otras actividades que lo ayudaría a enfrentar las cosas" $(\mathrm{M}=2.40)$, que pertenecen a estrategias de evitación en las dimensiones cognitiva y conductual, respectivamente. La persona presenta determinación para enfrentar dificultades asociadas al divorcio; sin embargo, esta determinación sería finita cuando, sumadas a esas vicisitudes, concomitan otras de tipo laboral, dada la misionalidad del uniformado.

En contraste, el personal civil que participó en el estudio, las tres más frecuentemente utilizadas son estrategias de aproximación en la dimensión conductual, "Rezó pidiendo ayuda o fuerza" $(\mathrm{M}=2.67)$, "Se esforzó tratando de hacer algo para que las cosas funcionaran" $(\mathrm{M}=2.39)$ y "Trató de aprender a hacer más cosas por sí mismo" $(\mathrm{M}=2.38)$. Esto da a entender que estas personas son determinadas para enfrentar los problemas relacionados con el divorcio, con acciones conducentes a disminuir el estrés de la situación (ver figura 3).

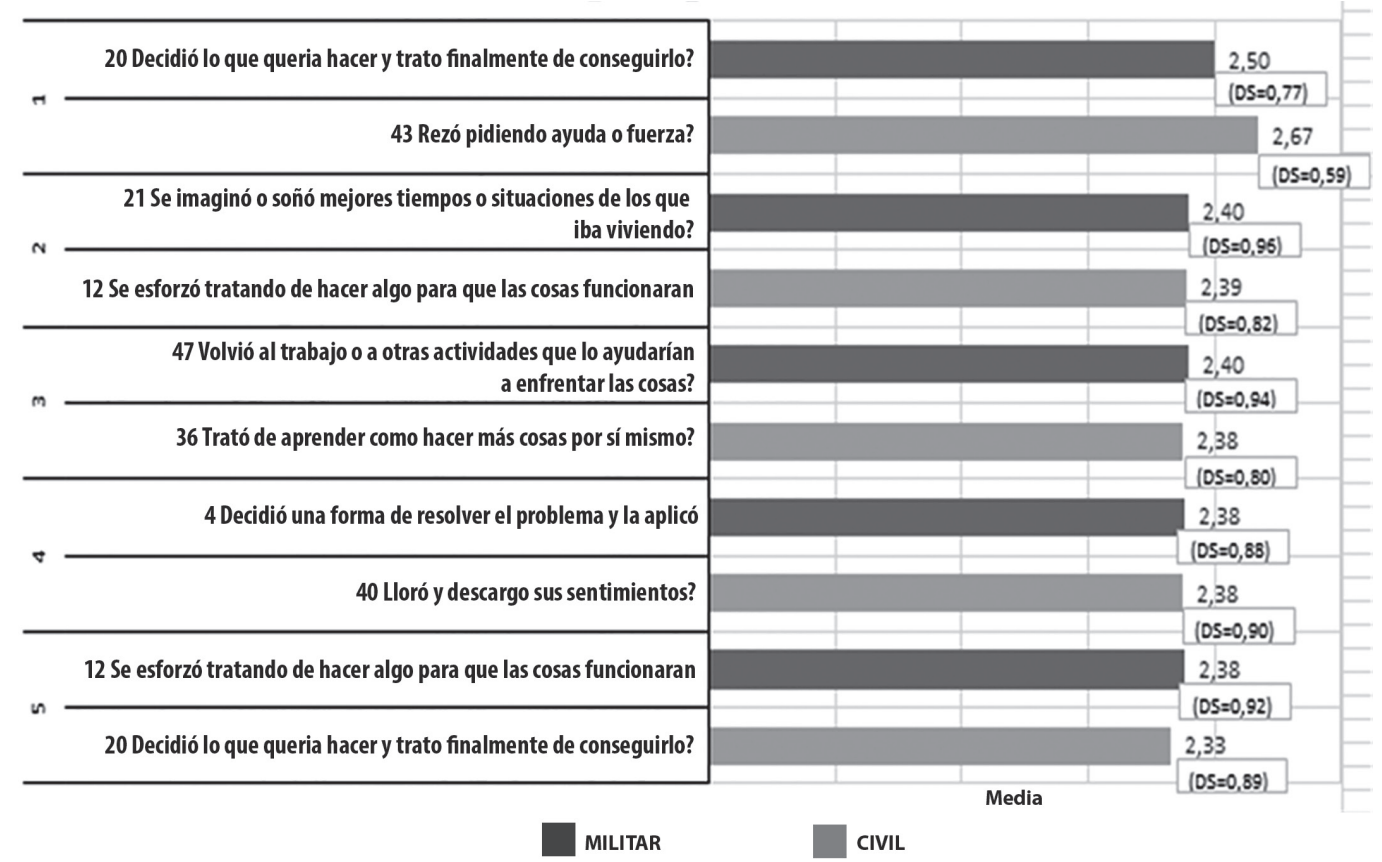

Figura 3. Estrategias de afrontamiento más usadas por civiles y militares.

Las estrategias de afrontamiento que menos utiliza el personal militar del estudio se reflejan en la evitación de conductas como: "Gritar como forma de desahogarse" (M=0.65), "Mantenerse alejados de la gente" ( $\mathrm{M=0.78)}$ las cuales son de evitación en la dimensión conductual, situación equiparable con el personal civil donde se presenta un comportamiento similar (ver figura 4). 


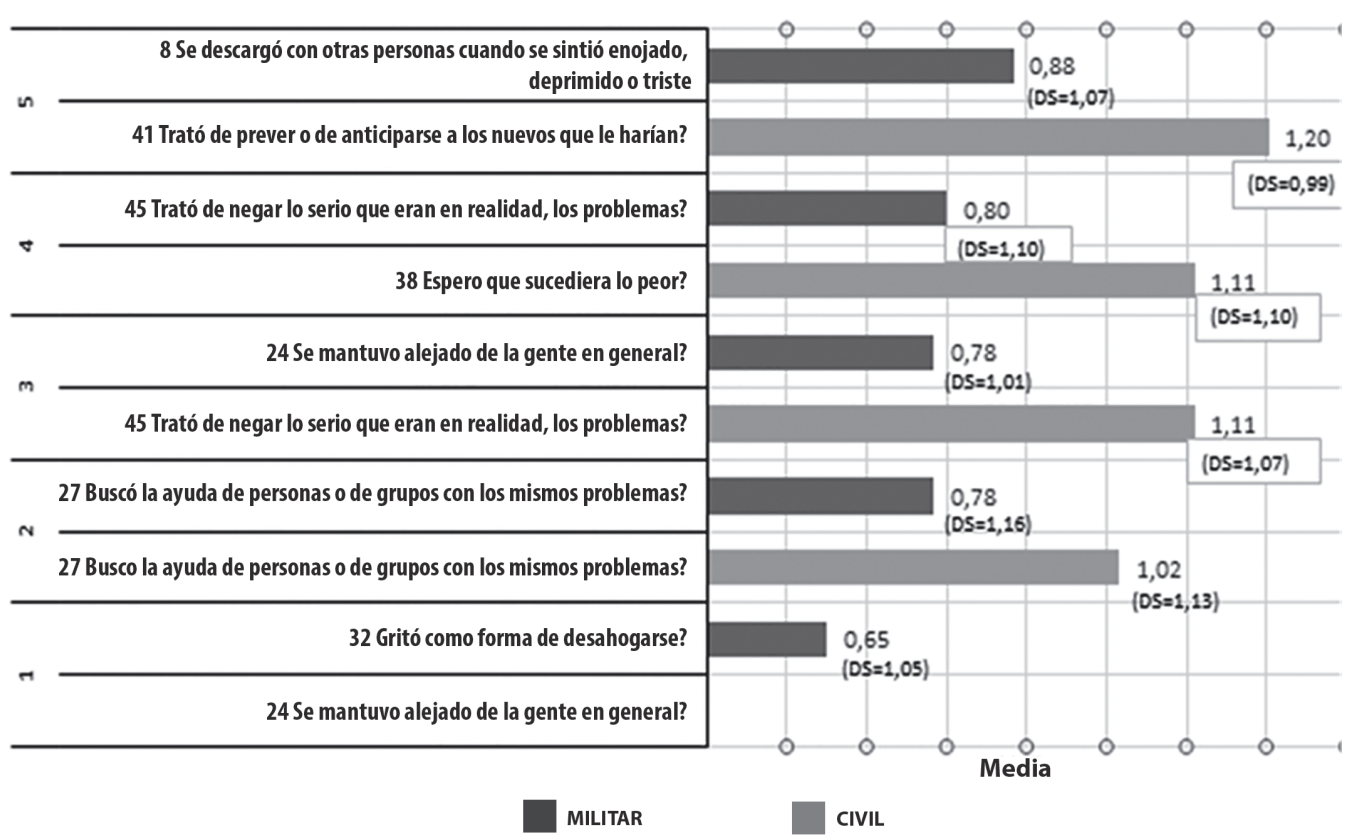

Figura 4. Estrategias de afrontamiento menos usadas por civiles y militares.

\section{Conclusiones}

El objetivo del presente estudio se enfocó en identificar cuáles son las estrategias de afrontamiento ante el divorcio o separación utilizadas por una muestra de población vinculada a las Fuerzas Armadas de Colombia, específicamente personal del Ejército Nacional y Armada Nacional, integrado por hombres y mujeres, con categoría de militares y civiles, quienes reportaron cuáles fueron las estrategias más y menos utilizadas ante la situación de marras. Este estudio contribuyó al análisis de los estilos de afrontamiento de las personas al momento de separarse.

La evidencia sugiere que no existirían estrategias funcionales o disfuncionales per se, sino que ello dependería de diversos factores, tales como las características del sujeto, sus recursos, y el ambiente (Endler, N. S. \& Parker, J. D. A., 1990, pp. 844-854). De esto se desprende que los hallazgos obtenidos sobre muestras generales podrían no ser aplicables a personas con distintos cuadros psicopatológicos (Vollrath, Markus \& Ribi, 2003, pp. 299-307).

Estos apartados de la literatura sugieren interpretar los resultados obtenidos, en el contexto en el cual se desarrolló el estudio, teniendo en cuenta aspectos propios de ambientes militares como alta tolerancia a la frustración, la habilidad para resolver conflictos, dada la naturaleza de su trabajo diario, así como, la plasticidad de los miembros de la familia para establecer nuevas redes de apoyo, en función a los traslados geográficos que experimentan los uniformados de manera periódica. 
Estrategias de afrontamiento frente al divorcio usadas por los participantes en general. En cuanto a las estrategias de afrontamiento más utilizadas por el personal participante del estudio e integrante de las Fuerzas Militares de Colombia, se encontró que la más predominante es la estrategia de aproximación en la dimensión conductual, lo que permite identificar en estas personas que, frente a la situación de divorcio, se focalizan en el problema, lo que refleja los esfuerzos cognitivos y conductuales para manejar o resolver los estresores vitales (Mikulic, I. \& Crespi, M., 2008, p. 307), como lo plantean Folkman, Lazarus, Dunkel-Schetter, DeLongis y Gruen (1986, pp. 992-1003). Centran sus esfuerzos cognitivos y conductuales en hacer frente a la situación, específicamente buscando guía y apoyo de carácter espiritual, ante la situación presentada.

Frente a la estrategia de afrontamiento "Decidió lo que quería hacer y trató firmemente de conseguirlo", de aproximación en la dimensión conductual, refleja en los participantes los niveles de determinación por resolver problemas, lo cual puede derivarse del entrenamiento del personal militar para resolver conflictos, variable que puede actuar como mediadora en la solución de problemas relacionados con el divorcio, siendo predictores de soluciones con más celeridad, y por ende, más bajos niveles de estrés.

Lo anterior demuestra que existen diferentes estrategias de afrontamiento que emplean las personas ante un divorcio, la literatura sugiere que éstas moderan los efectos negativos de experiencias de un evento traumático (Zukerman, G. \& Korn, L., 2013, pp. 1676-1690).

Dentro de las estrategias de afrontamiento menos utilizadas en las Fuerzas Militares se identificaron las de evitación, en la dimensión conductual, siendo la descarga emocional la menos empleada, entendida esta en los intentos conductuales de reducir la tensión expresando sentimientos negativos (Mikulic, I. \& Crespi, M., 2008, p. 307). Esto evidencia el nivel de regulación y autocontrol emocional de la población, para afrontar situaciones con altos niveles de estrés y tensión, en razón al entrenamiento que esta población recibe para manejar situaciones bajo presión.

En el mismo sentido, con respecto a la baja utilización de la escala búsqueda de apoyo, como estrategia de afrontamiento, la literatura muestra que esto constituye una desventaja frente a una situación de alto estrés como el divorcio. En concordancia, algunos autores encontraron que la búsqueda de apoyo social se relaciona con el descenso de síntomas depresivos (Veiel, H., Kuhner, C., Brill, G. \& Ihle, W., 1992, pp. 415-427), y también en los de tipo ansioso (Vollrath, M., Alnaes, R. \& Torgersen, S., 1994, pp. 727-736). Esta situación se presenta probablemente a causa de la alta movilidad de las familias de su sitio de vivienda, con la eventual falta de una red de apoyo, con la cual se puedan manejar situaciones tan íntimas como la ocurrencia de un divorcio.

Estrategias de afrontamiento frente al divorcio usadas en razón del sexo. Los resultados encontrados en cuanto a las estrategias de afrontamiento más y menos utilizadas en razón a los géneros hombre y mujer, coinciden con los estudios previos de Jiménez (2003, pp. 1-16) que señalan, además, que existen factores asociados al género, los cuales influyen en la manera en que las personas afrontan el proceso de separación. En el presente estudio estos matices se reflejan en la mayor utilización de estrategias de aproximación por parte de los hombres, en contraste con las mujeres, en quienes predominan las de evitación. 
En este sentido, las estrategias de afrontamiento más utilizadas por los hombres son las de aproximación en la dimensión conductual, reflejadas en las escalas solución de problemas y búsqueda de guía y apoyo, lo que coincide con lo planteado por Kunz (1996, pp. 3-4), quien en uno de sus estudios identificó la influencia de la familia de origen y red social en procesos de divorcio, concluyendo que la participación en reuniones, clubes y organizaciones, se relacionó significativamente con la sensación de que las cosas estarían mejor. Frente a la ocurrencia del divorcio, en el mismo sentido, también se sabe que los procesos de afrontamiento familiar que ilustran una dinámica de grupo, buscan regular las tensiones que enfrentan y, de este modo, garantizar la salud de sus miembros (McCubbin \& McCubbin, 1997, pp. 2-11)

Con respecto a las estrategias empleadas por las mujeres, los resultados indican que predominan las de evitación, tanto en la dimensión conductual como cognitiva, reflejadas en las escalas aceptación y resignación, evitación cognitiva y especialmente descarga emocional, siendo esta última la más empleada, en contraste con los hombres, por quienes esta escala es una de los menos empleada.

Para el caso de las estrategias menos empleadas por la mujeres, los resultados mostraron que, de igual manera, las estrategias de evitación en la dimensión conductual son las menos utilizadas, reflejado en estrategias como la descarga emocional, la búsqueda de guía y apoyo, la evitación cognitiva y la aceptación y resignación. En este orden de ideas, al analizar las estrategias de afrontamiento que utilizan los miembros de la pareja durante la separación, y los recursos psicológicos que mediatizan este proceso, es necesario cuestionar creencias asociadas a la "masculinidad" y "feminidad" avaladas por la cultura, entre las cuales se encuentran: la insensibilidad afectiva de los varones y la sensibilidad emocional de las mujeres (Valdés, Y., 2003, pp.1-16).

Estrategias de afrontamiento frente al divorcio usadas en razón de ser militar o civil. En cuanto a las estrategias de afrontamiento más utilizadas por el personal militar y civil, los resultados mostraron que en el personal militar predominan las estrategias de aproximación en la dimensión conductual, en la solución de problemas, lo que reflejó intentos conductuales de los militares participantes, por realizar acciones conducentes directamente a solucionar el problema (Mikulic, I. \& Crespi, M., 2008, p. 307). Esta situación es consonante con el estilo de vida de ellos, dado su entrenamiento militar y la misión relacionada con la lucha castrense.

Sin embargo y en contraste, los siguientes resultados encontrados en los militares, obedecen a estrategias de evitación en las dimensiones cognitiva y conductual respectivamente, presentes en escalas como evitación cognitiva y búsqueda de recompensas alternativas, lo que reflejó intentos cognitivos de evitar pensar en el problema de forma realista o intentos conductuales de involucrarse en actividades substitutivas y crear nuevas fuentes de satisfacción (Mikulic, I. \& Crespi, M., 2008, p. 307).

En este sentido, Holahan, Moos y Brennan, (1997) demostraron que las respuestas de afrontamiento varían en relación a la severidad del estresor: a mayor cantidad de sucesos de vida negativos y estresores crónicos, menor uso de respuestas por aproximación al problema, y más uso de respuestas por evitación, lo que llevó a pensar que los militares del estudio optaron por acciones o pensamientos evitativos, a raíz de la saturación de estresores de su vida diaria. 
Este hallazgo puede convertirse en un desafío investigativo para futuros procesos, se puede decir, fundamentados en la experiencia en temas de familia militar de los investigadores del presente estudio que, por razones relacionadas con la misión del uniformado, desafortunadamente en múltiples ocasiones los temas familiares y, específicamente los relacionados con el divorcio, deben ser dejados de lado a causa de situaciones como la lejanía geográfica, o la dificultad en encontrar apoyo, en cuanto a tiempos o permisos para resolver temas de tipo íntimo-familiar. Esto puede llevar a estas personas a tratar de evitar pensar o actuar frente al divorcio, por una imposibilidad manifiesta para hacerlo.

Resulta particular el anterior análisis, en la medida en que, según el imaginario colectivo, se espera que el militar reaccione de manera frontal ante un estresor, en este caso el divorcio. Sin embargo estos estudios demuestran, que por la saturación de estresores se pueden asumir cogniciones y conductas contrarias como la reflejada en el segundo puntaje de las estrategias de afrontamiento más frecuentes en militares.

Relacionado con lo anterior, llama la atención lo que muestra la comparación global de las tres estrategias de afrontamiento más utilizadas por militares y civiles, siendo estos últimos, a nivel general, las personas que más utilizan estrategias de aproximación a nivel conductual, presentes en escalas como como búsqueda de apoyo, solución de problemas y descarga emocional, frente a los militares, que si bien reportan la frecuencia más alta en estrategias de aproximación en la dimensión conductual; las dos siguientes frecuencias, pertenecen a estrategias de evitación, en las dimensiones cognitiva y conductual respectivamente, en escalas como evitación cognitiva y búsqueda de recompensas alternativas.

Lo dicho podría explicarse por el contexto en el cual se desarrolló el estudio, puesto que los civiles, en su mayoría mujeres, son las ex-esposas de los militares, quienes, por la particularidad ya citada de la familia militar, son quienes históricamente han tenido que tomar decisiones a nivel familiar, y en este caso con respecto al divorcio.

Respecto a las estrategias de afrontamiento menos empleadas por el personal militar, en los resultados se encontró que son las de evitación en la dimensión conductual. Estas estrategias de afrontamiento, también denominadas centradas en la emoción (Moos, R. H. \& Holahan, C. J., 2003, pp. 1387-1403), denotan la búsqueda de la regulación de las consecuencias emocionales activadas por la presencia de la situación estresante. Estos resultados se reflejan en la evitación por parte de los militares de usar acciones como: gritar como manera de desahogarse, o mantenerse alejado de la gente, situaciones que en el medio laboral podrían denotar debilidad o falta de carácter, especialmente cuando éstas pertenecen al ámbito íntimo-familiar de la persona.

Para el caso del personal civil, las estrategias menos empleadas coinciden con lo encontrado para el personal militar, siendo predominantes las de evitación en la dimensión conductual, en escalas como descarga emocional y evitación cognitiva; aquí también juega un papel importante el autocontrol y la regulación emocional, lo que mostró en este sentido que no hay una diferencia importante entre civiles y militares al momento de enfrentar una situación de divorcio.

Es de resaltar que entre las escalas menos utilizadas en el presente estudio, se encuentra la búsqueda de Orientación y apoyo, para militares y civiles; en conductas como la de buscar ayuda proveniente de personas, profesionales o grupos con los mismos problemas. Estos resultados no coinciden con lo expuesto por Kunz (1996, pp. 3-4), quien concluyó que la participación en 
reuniones, organizaciones y clubes estaba significativamente relacionada con la percepción de bienestar frente a la ocurrencia del divorcio.

Este resultado se puede interpretar a través de varias hipótesis como: alto nivel de reserva comunicativa del personal civil y militar que conforman las familias militares; poco tiempo de estas personas para atender situaciones relacionadas con el divorcio a través de búsqueda de apoyo en terceros y/o usuarios que no utilizan los servicios institucionales para la atención de la familia.

Teniendo en cuenta lo descrito, se puede concluir que estos resultados sugieren ser interpretados en el contexto en el cual se desarrolló el estudio, teniendo en cuenta aspectos propios de ambientes militares como alta tolerancia a la frustración; habilidad para resolver conflictos, debido a la naturaleza del trabajo diario y la exposición permanente a eventos altamente estresantes, así como la flexibilidad de los miembros de la familia para establecer nuevas redes de apoyo, en función a los traslados geográficos que experimentan los uniformados de manera periódica y factores de orden contextual y cultural.

Así mismo, que las estrategias de afrontamiento empleadas por las personas ante una situación de divorcio o separación inciden en el impacto psicosocial, en razón a las observaciones clínicas y los resultados de investigaciones sobre el divorcio, que sugieren que este es un evento traumático, así mismo en la necesidad de identificar, cómo las personas pueden hacer frente a un trauma, mediante la utilización de los repertorios conductuales, familiares, elementos que se han identificado en abundancia, como factores protectores, en familias con particularidades como las pertenecientes al ámbito militar.

Finalmente, la investigación revela consistentemente que el divorcio en general, en caso de ser difícil o destructivo, requiere de un tratamiento dirigido a la coparentalidad de los excónyuges y a la consecuente reorganización de las relaciones recíprocas de estos y con sus hijos, donde se priorice el bienestar de los hijos, además de controlar la violencia.

Para el caso de los "divorcios constructivos", se debe centrar la atención en el estrés que surge (descuido de las responsabilidades parentales, disputas desenfrenadas, reclutamiento de los hijos buscando que tomen partido). Pero también se debe trabajar la aceptación del fin del matrimonio, en busca de una relación funcional post-divorcio, ajuste emocional y cognitivo, un apoyo social, ajuste parental, además de una adaptación a la vida y bienestar físico.

\section{Recomendaciones}

La enriquecedora experiencia alcanzada en este trabajo sugiere la necesidad, para los investigadores interesados en el tema, de hacer estudios similares frente a otras experiencias traumáticas inherentes a los medios militares, tales como la muerte de un ser querido, los cambios familiares a causa de una herida de guerra con disminuciones físicas o cognitivas, secuestro por razones de guerra, privación de la libertad por motivos misionales, nacimiento de un hijo en condición de discapacidad o adaptación de un militar a la vida civil, después de una vida como uniformado; toda vez que estas son condiciones que se abordan permanentemente en las jefaturas de familia de la Fuerzas Militares, a través de programas de promoción y prevención. 
Diseñar investigaciones frente al problema planteado por Mikulic y Crespi (2008, pp. 305312), a causa de la falta de consenso respecto de qué tipo de estrategias de afrontamiento resultan óptimas frente al estrés; así como a la funcionalidad y la eficacia de los procesos de afrontamiento en el plano de la salud y el bienestar psicológico de los sujetos, en este caso para los miembros de las familias pertenecientes a las Fuerzas Militares.

A través de ejercicios similares, dar respuesta a la necesidad de analizar las modalidades de afrontamiento en grupos de sujetos que se hallan ante estresores específicos (Carver, C. et al., 1993, pp. 375-390; Folkman, et al., 1986, pp. 992-1003), como es el caso de la población militar.

Estudiar fenómenos emergentes en el proceso de reflexión como: altos nivel de reserva comunicativa del personal civil y militar que conforman las familias militares, en especial frente a fenómenos como el divorcio; subregistro en la ocurrencia de divorcios, o simplemente que los usuarios no utilicen a los servicios institucionales para la atención de la familia.

\section{Referencias}

1. Aldous, J. \& Ganey, R. (1999). "Family life and the pursuit of happiness: The influence of gender and race". Journal of Family Issues, 20, 155-180.

2. Alyssa, J, Jay, S., Stephen, W., Bradley, N., Joseph, P. \& Charles, C. (2010). "Deployment and the use of mental health services among U.S. Army wives". The New England Journal of Medicine. Vol. 362 No. 2 (january 14, 2010), pp. 101-109.

3. Amarís, M. Madariaga, Valle \& Zambrano (2013). "Estrategias de afrontamiento individual y familiar frente a situaciones de estrés psicológico". Psicología desde el Caribe, vol. $30, \mathrm{~N} .^{\circ} 1$, enero-abril, pp. 123 - 145. Barranquilla, Colombia: Universidad del Norte.

4. Aseltine Jr, R. H., \& Kessler, R. C. (1993). "Marital disruption and depression in a community sample". Journal of Health and Social Behavior, 237-251.Recuperado de: http://www.jstor.org/stable/2137205?seq=1\#page_scan_ tab_contents

5. Carver, C. et al. (1993). "How coping mediates the effect of optimism on distress: A study of women with early stage breast cancer". Journal of Personality and Social Psychology, 65, 375-390.

6. Ciro, A. (2014). Transformación estructural del Ejército colombiano. Revista Científica "General José María Córdova". $12(13), 19-88$.
7. De Zubiría, M. (2011) "Ser Mejores Padres I: Conozco el desarrollo afectivo de mis hijos". Bogotá, Colombia: Fundación Internacional de Pedagogía Conceptual Alberto Merani. Recuperado de: https://centrodeformacionafectiva.wordpress.com/tag/miguel-de-zubiria/

8. DIFAM (2014). Estadísticas institucionales. Bogotá, Colombia: Dirección de Familia y Asistencia del Ejército Nacional de Colombia.

9. Endler, N.S., \& Parker, J.D.A. (1990). "Multidimensional assessment of coping: A critical evaluation". Journal of Personality and Social Psychology, 58, 844-854.

10. Folkman, S., Lazarus, R. S., Dunkel-Schetter, C., DeLongis, A., \& Gruen, R. J. (1986). "Dynamics of a stressful encounter: cognitive appraisal, coping, and encounter outcomes". Journal of personality and social psychology, 50(5), 992-1003.

11. Gager, C. \& Hohmann-Marriott, B. (2006). "Distributive justice in the household: A comparison of alternative theoretical models". Marriage \& Family Review, 40(2-3), 5-42.

12. García M. (2003) El cuestionario como instrumento de investigación/evaluación. Almendralejo. Recuperado de: http://cvonline.uaeh.edu.mx/Cursos/Maestria/MTE/ Gen02/seminario_de_tesis/Unidad_4_anterior/Lect_El_ Cuestionario.pdf

13. Holahan C., Moos R. \& Brennan P. (1997) Social context, coping strategies, and depresive symptoms: an expanded model 
with cardiac patients. 72(4):918-28. Recuperado de: www. ncbi.nlm.nih.gov/pubmed/9108704

14. Jimenez, M. M., Macías, M. A., \& Amaris, M. V. (2012). Afrontamiento en crisis familiares. Salud Uninorte, 28(1).

15. Jiménez, Y. V. (2003) Impacto psicológico del divorcio en la mujer. Una nueva visión de un viejo problema. Recuperado de: http://biblioteca.clacso.edu.ar/Cuba/cips/20120823041915/ valdes1.pdf

16. Kunz (1996). "Social support during the process of divorce". Journal of divorce \& remarriage, volumen 24, issue 3-4.

17. Lippman, L., Wilcox, B. \& Ryberg, R. Mapa mundial de la familia (2013). Los cambios en la familia y su impacto en el bienestar de la niñez. Child Trends. Recuperado de: http://www.childtrends.org/wpcontent/uploads/2013/05/ Mapa_mundial_familia_2013.pdf

18. Martínez, C. (2013). Encuesta Nacional de Demografía y Salud, ENDS, 1990 / 2010. Descenso de la fecundidad, bono demográfico y crecimiento económico en Colombia. 1990-2010.

Recuperado de: https://www.minsalud.gov.co/sites/rid/Lists/ BibliotecaDigital/RIDE/INEC/INV/0\%20-\%20SERIE\%20 ESTUDIOS\%20A\%20PROFUNDIDAD\%20FINAL\%20 WEB\%202014.pdf

19. McCubbin, H.I., M.A. McCubbin, A.I. Thompson, S.V. Han, and C.T. Allen (1997). Families under stress: what makes them resilient. Journal of Family and Consumer Sciences, 89, 2-11.

20. Mikulic, I. \& Crespi, M. (2005). Ecological transition": a study of coping with migration, imprisonment and divorce life crisis from positive psychology approach. En: Anuario de investigaciones, vol. XIII. Buenos Aires, Argentina: Facultad de Psicología, Universidad de Buenos Aires, UBA, Secretaría de investigaciones.

21. Mikulic, I. \& Crespi, M. (2008). "Adaptación y validación del inventario de respuesta de afrontamiento de Moss
CRI-A para adultos". Anuario de Investigaciones, 15(2), 305-312.

22. Ojeda N. \& González E. (2008). "Divorcio y separación conyugal en México en los albores del siglo XXI". Revista Mexicana de Sociología, Vol. 70, N. 1 (Enero-marzo), pp. $111-145$

23. Superintendencia de Notariado y Registro (2013). Estadísticas institucionales. Bogotá, Colombia.

24. Valdés Y. (2003). Impacto psicológico del divorcio en la mujer. Una nueva visión de un viejo problema. Recuperado de: http://biblioteca.clacso.edu.ar/Cuba/cips/20120823041915/ valdes1.pdf

25. Veiel H., Kuhner C., Brill G., \& Ihle W. (1992). "Psycosocial correlates of clinical depression after Psychiatric in-patient treadment: Methodological issues and baseline differences between recovered and non-recovered patients". Psycological Medicine, 22, 415-427.

26. Vollrath M., Alnaes R., \& Torgersen S. (1994). Coping and MCMI-II symptom scales. Journal of clinical psycology, 50, 727-736.

27. Vollrath M. Markus A. \& Ribi K. (2003). "Personality of children with Accident-related injuries". European Journal of Personality. 17, 299-307.

28. Yárnoz-Yaben, S. (2013). "Perdón y divorcio: una perspectiva clínica”. Clínica, 4(3), 211-221.

29. Zimmerman C. \& Uranga E. (1946) "El problema básico de la familia”. Revista Mexicana de Sociología, Vol. 8 No. 2 recuperado de: http://www.jstor.org.ezproxy.unisabana. edu.co/stable/pdfplus/10.2307/3537266.pdf

30. Zukerman, G. \& Korn L. (2013) Post-traumatic stress and world assumptions: The effects of religious coping. Recuperado de: http:// link.springer.com/article/10.1007\%2Fs10943-013-9755-5\#page-1 